\title{
Cirurgia colorretal videolaparoscópica: experiência inicial na abordagem de 90 pacientes, no Programa de Pós-graduação sensu lato (residência e especialização) em Coloproctologia, pelo Grupo de Coloproctologia de Belo Horizonte
}

\author{
Videolaparoscopic colorectal surgery: initial report of surgical approach of 90 patients in \\ a Postgraduate Program (residency) in Coloproctology by the Group of Coloproctology \\ of Belo Horizonte
}

\begin{abstract}
ANTONIO CARLOS BARROS LIMA JUNIOR ${ }^{1}$, FABIO GONTIJO RODRIGUES ${ }^{1}$, GUILHERME DE ALMEIDA SANTOS ${ }^{1}$, CAROLINE PINTO COUTINHO ${ }^{1}$, ESTEVAN GUILLERMO VIGIL VERASTEGUI SILVA ${ }^{2}$, PETERSON MARTINS NEVES ${ }^{3}$, JOSÉ ROBERTO MONTEIRO CONSTANTINO ${ }^{3}$, ÁUREA CÁSSIA GUALBETO BRAGA ${ }^{3}$, RENATA MAGALI RIBEIRO

SILLUZIO FERREIRA ${ }^{3}$, ISABELLA MENDONÇAALVARENGA³, DAVID DE LANNA ${ }^{3}$, RICARDO GUIMARÃES TEIXEIRA ${ }^{3}$, HERALDO NEVES VALLE JUNIOR ${ }^{3}$, SINARA MÔNICA OLIVEIRA LEITE ${ }^{3}$, LUCIANA MARIA PYRAMO COSTA $^{3}$, ILSON GERALDO DA SILVA ${ }^{4}$, GERALDO MAGELA GOMES DA CRUZ ${ }^{5}$
\end{abstract}

\begin{abstract}
${ }^{1}$ Residentes do Grupo de Coloproctologia da Santa Casa de Belo Horizonte e Faculdade de Ciências Médicas de Minas Gerais Minas Gerais (MG), Brasil. ${ }^{2}$ Estagiário voluntário do Grupo de Coloproctologia da Santa Casa de Belo Horizonte e Faculdade de Ciências Médicas de Minas Gerais - Minas Gerais (MG), Brasil. ${ }^{3}$ Assistentes efetivos do Grupo de Coloproctologia da Santa Casa de Belo Horizonte e Faculdade de Ciências Médicas de Minas Gerais-Minas Gerais (MG), Brasil. ${ }^{4}$ Chefe Interino do Serviço de Coloproctologia de Homens da Santa Casa de Belo Horizonte-Minas Gerais (MG), Brasil. ${ }^{5}$ Chefe do Serviço de Coloproctologia de Mulheres e do Ambulatório de Coloproctologia da Santa Casa de Belo Horizonte-Minas Gerais (MG), Brasil; Coordenador do Grupo de Coloproctologia da Santa Casa de Belo Horizonte e Faculdade de Ciências Médicas de Minas Gerais-Minas Gerais (MG), Brasil.
\end{abstract}

JUNIOR ACBL, RODRIGUES FG, SANTOS GDA, COUTINHO CP, SILVA EGVV, NEVES PM, CONSTANTINO JRM, BRAGA ÁCG, FERREIRA RMRS, ALVARENGA IM, LANNA DD, TEIXEIRA RG, JUNIOR HNV, LEITE SMO, COSTA LMP, SILVA IGD, CRUZ GMGD. Cirurgia colorretal videolaparoscópica: experiência inicial na abordagem de 90 pacientes, no Programa de Pós-graduação sensu lato (residência e especialização) em Coloproctologia, pelo Grupo de Coloproctologia de Belo Horizonte. Rev bras Coloproct, 2011;31(2): 169-183.

RESUMO: O objetivo do trabalho foi proceder a uma revisão criteriosa de uma casuística de 90 pacientes submetidos às ressecções colorretais por via videolaparoscópica no decurso de 12 meses (maio de 2009 a maio de 2010). A média etária foi de 62,1 anos, com extremos de 20 e 93 anos, sendo a maioria dos pacientes do sexo feminino $(52 ; 57,8 \%)$. O diagnóstico mais comum foi câncer colorretal, com 60 casos (66,7\%), seguido pelos pólipos colorretais, com 12 casos $(13,4 \%)$, doença diverticular, com sete casos (7,8\%), e outros diagnósticos, com 11 casos $(\mathbf{1 2 , 1 \% )}$. O preparo intestinal mais realizado foi com o Picolax (53 casos; 58,9\%); 76 pacientes foram submetidos à colonoscopia e biópsia (84,4\%). As cirurgias mais realizadas foram as retossigmoidectomias (54 casos; 60,0\%), seguidas pelas hemicolectomias direitas (20 casos; 22,2\%). O tempo de execução da maioria das cirurgias foi entre duas e três horas (34 casos; 37,8\%) e entre três e quatro horas ( 24 casos, 26,7\%), com média de 203 minutos. Em 81 casos, houve anastomoses (90,0\%), a maioria mecânica intra-abdominal (55,6\%) e manual extra-abdominal ( 25 casos; $27,8 \%$ ), tendo sido o conjunto de grampeadores circulares e lineares articulados os recursos mais utilizados ( 50 casos; $55,6 \%$ ). O eletrocautério foi usado em 68 pacientes $(\mathbf{7 5 , 6 \%})$. A incisão abdominal mais usada foi a de Mallard (39 casos, $43,4 \%$ ) e a mediana ( 22 casos; $24,4 \%$ ), sendo as extensões mais comuns entre 6 e $10 \mathrm{~cm}$ (55 casos; 78,6\%). Houve 12 intercorrências peroperatórias (13,2\%), que levaram a conversões para laparotomia. A média de dimensões das peças cirúrgicas foi 33,2 cm, a maioria entre 21 e $30 \mathrm{~cm}$ (51 casos, 56,8\%). Houve 13 complicações pós-operatórias (14,4\%), 11 delas cirúrgicas (12,2\%) e duas clínicas $(2,2 \%)$, das quais decorreram três óbitos, sendo dois cirúrgicos e um clínico. O tempo médio de internamento foi de 5,3 dias, tendo sido 57 (63,3\%) a até cinco dias. Foram para o CTI 28 pacientes (31,1\%), sobretudo por conta de comorbidades (22 casos; 24,4\%). A liberação de dieta oral foi de um dia para 49 pacientes $(54,5 \%)$. Os autores comparam os resultados com a bibliografia correlata.

Palavras-chave: cirurgia colorretal; laparoscopia.

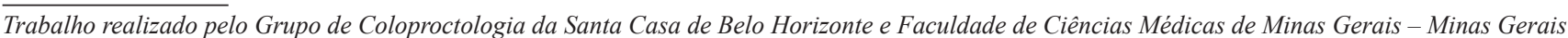
$(M G)$, Brasil.

Recebido em: 05/10/2010

Aprovado em: 27/10/2010 
Rev bras Coloproct Abril/Junho, 2011
Cirurgia colorretal videolaparoscópica: experiência inicial na abordagem de 90 pacientes, no Programa de Pós-graduação sensu lato (residência e especialização) em Coloproctologia, pelo Grupo de Coloproctologia de Belo Horizonte Antonio Carlos Barros Lima Junior e Cols.
Vol. 31

$\mathbf{N}^{\circ} 2$

\section{INTRODUÇÃO}

Os procedimentos cirúrgicos por videolaparoscopia são realizados, atualmente, como rotina em muitos serviços tal a experiência e a segurança acumuladas ao longo dos últimos 10 a 20 anos, não havendo dúvidas de que acarretam uma melhor resposta ao trauma, tanto sob o ponto de vista doloroso quanto inflamatório, endócrino e metabólico, quando comparados aos procedimentos laparotômicos. Todavia, é técnica que se reveste de características próprias e extremamente peculiares, tanto no tocante ao treinamento dos cirurgiões quanto aos recursos alocados e aspectos relacionados à sua prática. Em decorrência disso, é comum que vários especialistas de um determinado serviço pratiquem a técnica em vários hospitais, enquanto o próprio serviço não o pratique em sua própria sede pelos motivos mais variados, o que inclui a indisponibilidade dos recursos necessários. Assim, embora vários especialistas do Grupo de Coloproctologia da Santa Casa de Belo Horizonte e Faculdade de Ciências Médicas de Minas Gerais (GCP) se dedicassem à cirurgia videolaparoscópica em outros hospitais, somente em maio de 2009 a técnica foi incorporada por todos os membros do Grupo e inserida na pós-graduação da especialidade na Santa Casa de Belo Horizonte.

Assim, dentro do Programa de Pós-graduação em Coloproctologia sensu lato, a partir do mês de maio de 2009, o GCP, após um período variado de aprendizagem e treinamento, iniciou a prática rotineira de cirurgia videolaparoscópica para abordagem das doenças colorretais, mesmo ainda em números muito inferiores que aos de cirurgias laparotômicas. Durante este período de 12 meses (maio de 2009 a maio de 2010), foram operados, pelo Grupo, por essa técnica, 90 pacientes, o que equivale a cerca de oito cirurgias videolaparoscópicas por mês, que corresponde a cerca de um quinto das cirurgias laparotômicas no mesmo período. Provavelmente, essa proporção deverá cair nos anos vindouros certamente em decorrência da diminuição dos critérios de exclusão atualmente usados - laparotomias prévias e complicações da doença abordada.

\section{OBJETIVO}

O objetivo do trabalho foi proceder a uma revisão criteriosa de uma casuística atual de 90 pacientes submetidos a ressecções colorretais por via videolaparoscópica no decurso de 12 meses (maio de 2009 a maio de 2010), com dois critérios de exclusão - laparotomias prévias e complicações da doença de base abordada.

\section{CASUÍSTICA - MATERIAIS E MÉTODOS}

Dentro do Programa de Pós-graduação em Coloproctologia sensu lato (Residência e Especialização), o Grupo de Coloproctologia da Santa Casa de Belo Horizonte e Faculdade de Ciências Médicas de Minas Gerais (GCP-SCBH-FCMMG), durante 12 meses (maio de 2009 a maio de 2010), operou, por via videolaparoscópica, 90 pacientes pelas mais variadas doenças. Foi criado um protocolo de abordagem dos pacientes contendo os seguintes dados, que foram minuciosamente revisados:

1. Idade dos pacientes.

2. Gênero dos pacientes.

3. Cirurgião e equipe cirúrgica do Grupo de Coloproctologia.

4. Diagnóstico principal - doença de base abordada pela cirurgia.

5. Resultado de colonoscopia e de exame histopatológico de biópsias realizadas.

6. Preparo intestinal para colonoscopia e cirurgia.

7. Tempo de realização de cirurgia.

8. Técnica cirúrgica realizada.

9. Tipo de anastomose (manual ou mecânica).

10. Tipo de material usado para corte e coagulação e dissecção.

11. Tipos de grampeadores utilizados (grampeadores circulares, grampeadores lineares e angulares cortantes).

12. Tipo de incisão abdominal para suturas e extração de peças cirúrgicas.

13. Intercorrências peroperatórias e suas abordagens.

14. Dimensões das peças cirúrgicas.

15. Conversões de cirurgias de vídeolaparoscopia para laparotomia (fases e motivos).

16. Complicações pós-operatórias e suas abordagens.

17. Período de hospitalização.

18. Internamento em CTI e duração.

19. Tempo (dias) de liberação de dieta oral.

20. Comorbidades apresentadas pelos pacientes. 
É importante ressaltar que foram adotados apenas dois critérios de exclusão: pacientes previamente laparotomizados e pacientes apresentando complicações da doença de base a ser abordada, ficando a decisão pela abordagem por via laparoscópica na dependência exclusiva de disponibilidade da aparelhagem no dia da cirurgia.

O presente projeto foi aprovado pelo Comitê de Ética da Santa Casa de Belo Horizonte.

\section{RESULTADOS}

\section{Idade e gênero}

A média etária dos pacientes foi 62,1 anos, com extremos de 20 e 93 anos, sendo estatisticamente significativas $(\mathrm{p}<0,05)$ as faixas de 51 a 60 anos $(27$ pacientes; $30,0 \%)$ e 61 a 70 anos (21 pacientes; $23,3 \%$ ) (Tabela 1 e Figura 1).

\section{Gênero}

Dos pacientes, 52 eram mulheres $(57,8 \%)$ e 38 eram homens $(42,2 \%)$ (Tabela 2 e Figura 2$)(p>0,05)$.

\section{Preparo intestinal}

Os preparos intestinais para colonoscopia e para cirurgia (o mesmo para um determinado paciente) foram os seguintes: 53 com Picolax $(58,9 \%), 22$ com Dufalac (24,4\%), 19 com Manitol (11,1\%) e cinco com Lactulose $(5,6 \%)$ (Tabela 3 e Figura 3 ).

\section{Colonoscopia e biópsia}

A colonoscopia foi realizada em 86 pacientes $(95,5 \%)$, dos quais 76 com biópsias para exame histopatológico $(84,4 \%)$ (Tabela 4 e Figura 4$).$

\section{Diagnósticos}

O diagnóstico mais comum foi câncer colorretal, com 60 casos $(66,7 \%)$, seguido pelos pólipos colorretais, com 12 casos (13,4\%), doença diverticular, com sete casos $(7,8 \%)$, e outros diagnósticos, com 11 casos (12,1\%) (Tabela 5 e Figura 5). Dos 60 casos de câncer colorretal, dez localizavam-se no reto baixo, dez no reto alto, seis no retossigmoide e 19 no sigmoide, totalizando 45 casos no reto e sigmoide; os demais tumores se localizaram no cólon descendente (um caso), cólon transverso (dois casos), ângulo hepá-
Tabela 1. Distribuição, por décadas etárias, de 90 pacientes submetidos a cirurgias colorretais por via videolaparoscópica: com média etária de 62,1 anos, a mínima de 20 anos e a máxima de 93 anos, a década que mais se sobressaiu foi a sexta década (51-60 anos), somando 27 pacientes (30,0\%), seguida imediatamente pela sétima década (61-70 anos) com 21 pacientes $(23,3 \%)$ (faixas etárias com $p<0,05)$.

\begin{tabular}{lcccc}
\hline Idades (anos) & $\mathbf{N}$ & $\mathbf{\%}$ & $\mathbf{\Sigma} \mathbf{N}$ & $\mathbf{\Sigma} \%$ \\
\hline$<10$ & 00 & 0 & 00 & 0 \\
$11-20$ & 01 & 1,1 & 01 & 1,1 \\
$21-30$ & 00 & 0 & 01 & 1,1 \\
$31-40$ & 09 & 10,0 & 10 & 11,1 \\
$41-50$ & 17 & 18,9 & 27 & 30,0 \\
$51-60$ & 27 & 30,0 & 54 & 60,0 \\
$61-70$ & 21 & 23,3 & 75 & 83,3 \\
$71-80$ & 14 & 15,6 & 89 & 98,9 \\
$81-90$ & 00 & 0 & 89 & 98,9 \\
$91-100$ & 01 & 1,1 & 90 & 100,0 \\
Total & 90 & 100,0 & 90 & 100,0 \\
\hline
\end{tabular}

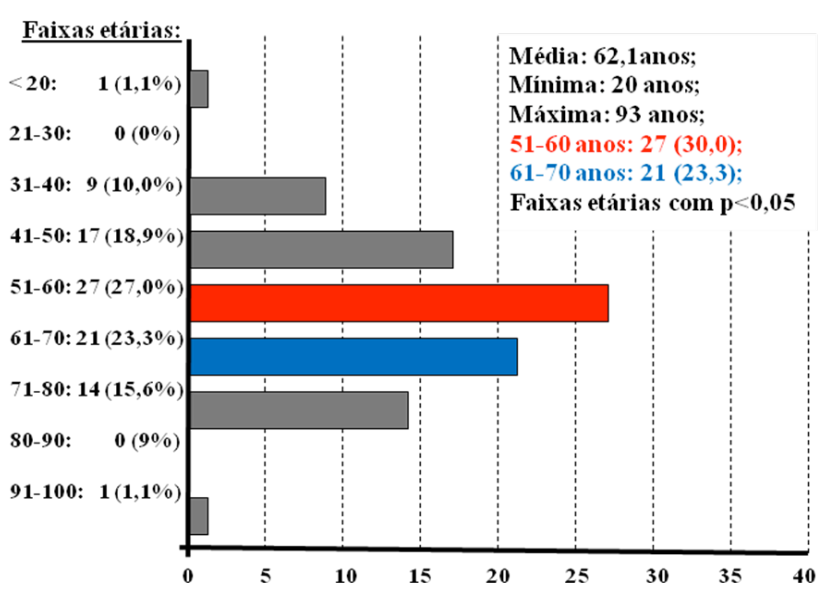

Figura 1. Distribuição, por décadas etárias, de 90 pacientes submetidos a cirurgias colorretais por via videolaparoscópica: chamam a atenção as colunas representando a sexta e sétima décadas, com 27 (30,0\%) e 21 pacientes (23,3\%) (faixas etárias com $p<0,05)$, totalizando 48 pacientes $(53,3 \%)$.

tico (um caso), cólon descendente (três casos) e ceco (oito casos). Os 11 casos de outros diagnósticos foram os seguintes: fístula retovaginal (um caso, $1,1 \%$ ), doença de Crohn de íleo terminal (um caso, 1,1\%), diverticulose colônica difusa (dois casos, 2,2\%), retocolite ulcerativa inespecífica com câncer de sigmoide (um caso, 1,1\%), endometriose (um caso, 1,1\%), megacólon chagasico (um caso, 1,1\%), doença de Crohn 
Tabela 2. Distribuição, por gêneros, de 90 pacientes submetidos a cirurgias colorretais por via videolaparoscópica: mulheres representaram 57,8\% (52 pacientes) e homens 42,2\% (38 pacientes).

\begin{tabular}{llr}
\hline Gêneros & $\mathbf{N}$ & $\mathbf{\%}$ \\
\hline Feminino & 52 & 57,8 \\
Masculino & 38 & 42,2 \\
Total & 90 & 100,0 \\
\hline
\end{tabular}

Gêneros:

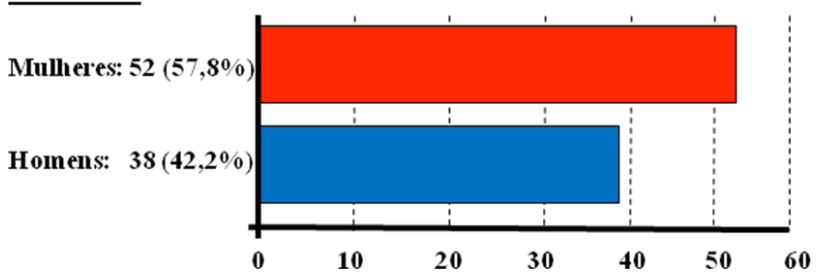

Figura 2. Distribuição, por gêneros, de 90 pacientes submetidos a cirurgias colorretais por via videolaparoscópica: mulheres representaram $57,8 \%$ (52 pacientes) e homens $42,2 \%$ (38 pacientes).

Tabela 3. Distribuição, por preparos intestinais, de 90 pacientes submetidos a cirurgias colorretais por via videolaparoscópica: o Picolax foi o medicamento mais usado no preparo intestinal, seja para as colonoscopias seja para as cirurgias (53 pacientes; $58,9 \%)(p<0,05)$, seguido de longe pelas demais formas de preparo intestinal.

\begin{tabular}{llr}
\hline Preparo intestinal & N & \% \\
\hline Picolax & 53 & 58,9 \\
Manitol & 10 & 11,1 \\
Lactulose & 05 & 5,6 \\
Dufalac & 22 & 24,4 \\
Total & 90 & 100,0 \\
\hline
\end{tabular}

\section{Preparointestinal:}

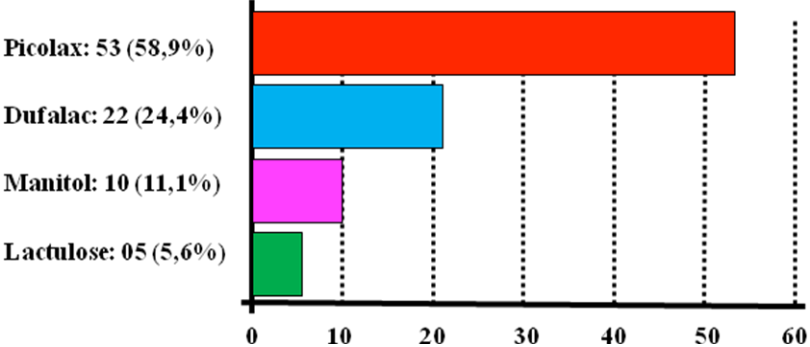

Figura 3. Distribuição, por preparos intestinais, de 90 pacientes submetidos a cirurgias colorretais por via videolaparoscópica: o Picolax foi o medicamento mais usado no preparo intestinal, seja para as colonoscopias seja para as cirurgias (53 pacientes; 58,9\%) $(p<0,05)$, seguido de longe pelas demais formas de preparo intestinal. de íleo terminal e períneo (um caso, 1,1\%), e pólipo ressecado previamente sem margem de segurança (um caso, 1,1\%) (Tabela 5 e Figura 5).

\section{Cirurgias realizadas}

Foram as seguintes as técnicas cirúrgicas praticadas nos 90 pacientes, por via laparoscópica: retossigmoidectomia abdominal com anastomose colorretal (RSA-ACR) em 54 pacientes $(60,0 \%)$, hemicolectomia direita com anastomose íleo-transverso (HCDAIT) em 20 pacientes $(22,2 \%)$, colectomia total com anastomose ileorretal (CT-AIR) em quatro pacientes (4,4\%), hemicolectomia esquerda com anastomose transversorretal (HCE-ATR) em dois pacientes (2,2\%), proctocolectomia total com ileostomia terminal (PCTIT) em um paciente $(1,1 \%)$, amputação abdôminoperineal com colostomia definitiva (AAP-CD) em cinco pacientes $(5,7 \%)$, ressecção local de endometriose em um paciente $(1,1 \%)$, Hartmann em um paciente $(1,1 \%)$, Duhamel em um paciente $(1,1 \%)$ e colostomia em um paciente $(1,1 \%)$ (Tabela 6 e Figura 6 ).

Tabela 4. Distribuição, por colonoscopias $e$ biópsias para exame histopatológicos, de 90 pacientes submetidos a cirurgias colorretais por via videolaparoscópica: a quase totalidade dos pacientes (76 pacientes; $84,4 \%$ ) foi submetida à colonoscopia e biópsia $(p<0,05)$.

\begin{tabular}{lcr}
\hline Colonoscopia e biópsia & N & \multicolumn{1}{c}{$\%$} \\
\hline Colonoscopia e biópsia & 76 & 84,4 \\
Colonoscopia sem biópsia & 10 & 11,1 \\
Sem colonoscopia & 04 & 4,5 \\
Total & 90 & 100,0 \\
\hline
\end{tabular}

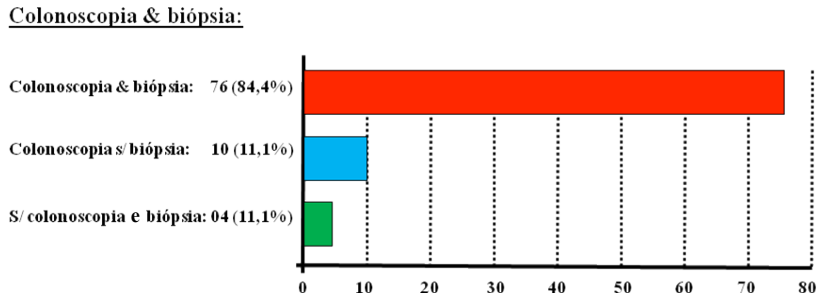

Figura 4. Distribuição, por colonoscopias e biópsias para exames histopatológicos, de 90 pacientes submetidos a cirurgias colorretais por via videolaparoscópica: a coluna representativa do número de pacientes que foram submetidos à colonoscopia com biópsia (76 pacientes; 84,4\%) comprova a significância estatística $(p<0,05)$. 
Tabela 5. Distribuição, por diagnósticos principais, de 90 pacientes submetidos a cirurgias colorretais por via videolaparoscópica: foi marcante a incidência de câncer colorretal (60 pacientes; 66,7\%) $(p<0,05)$, secundado pelos pólipos colorretais.

\begin{tabular}{lcc}
\hline Diagnósticos principais & N & \% \\
\hline Câncer colorretal & 60 & 66,7 \\
Câncer de reto baixo & 10 & \\
Câncer de reto alto & 10 & \\
Câncer de retossigmoide & 06 & \\
Câncer de sigmoide & 19 & \\
Câncer de cólon descendente & 01 & \\
Câncer de cólon transverso & 02 & \\
Câncer de ângulo hepático & 01 & \\
Câncer de cólon ascendente & 03 & \\
Câncer de ceco & 08 & \\
Pólipos e tubulares e vilosos & 12 & 13,4 \\
Doença diverticular de sigmoide & 07 & 7,8 \\
Fístula retovaginal (RT) & 01 & 1,1 \\
Doença de Crohn íleo terminal & 01 & 1,1 \\
Diverticulose colônica difusa & 02 & 2,2 \\
FAP & 02 & 2,2 \\
RCUI e câncer de sigmoide & 01 & 1,1 \\
Endometriose & 01 & 1,1 \\
Megacólon chagásico & 01 & 1,1 \\
Doença de Crohn de íleo e períneo & 01 & 1,1 \\
Pólipo ressecado sem margem & 01 & 1,1 \\
TOTAL & 90 & 100,0 \\
\hline
\end{tabular}

Diagnósticos principais:

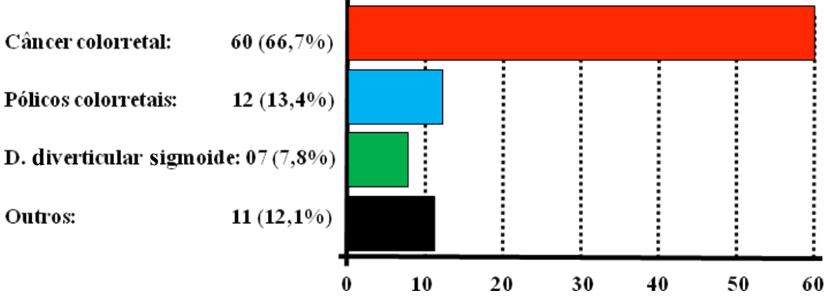

Figura 5. Distribuição, por diagnósticos principais, de90pacientes submetidos a cirurgias colorretais por via videolaparoscópica: é marcante o significado estatístico da incidência de câncer colorretal representado pela primeira coluna (60 pacientes; $66,7 \%$ ) ( $p<0,05)$, secundado pelos pólipos colorretais.
Tabela 6. Distribuição, por cirurgias realizadas, de 90 pacientes submetidos a cirurgias colorretais por via videolaparoscópica: em decorrência de a elevada incidência de doenças cirúrgicas colorretais se localizarem distalmente no intestino grosso, as cirurgias mais comuns foram as retossigmoidectomias abdominais com anastomose colorretal (54 casos; 60,0\%) ( $p<0,05)$, seguidas, de longe, pelas hemicolectomias direitas com anastomose ileo-transverso (20 casos; 22,2\%).

\begin{tabular}{lcr}
\hline Cirurgias colorretais & N & $\mathbf{0} \%$ \\
\hline RSA-ACRR & 54 & 60,0 \\
HCD-AIT & 20 & 22,2 \\
AAP-CT & 05 & 5,7 \\
CT-AIR & 04 & 4,4 \\
HCE-ATR & 02 & 2,2 \\
PCT-IT & 01 & 1,1 \\
Hartmann & 01 & 1,1 \\
Duhamell & 01 & 1,1 \\
Colostomia & 01 & 1,1 \\
Ressecção local de endometriose & 01 & 1,1 \\
TOTAL & 90 & 100,0 \\
\hline
\end{tabular}

Siglas: RSA-ACR: Retossigmoidectomia abdominal com anastomose colorretal; HCD-AIT: Hemicolectomia direita com anastomose íleotransverso; CT-AIR: Colectomia total com anastomose ileo-retal; HCE-ATR: Hemicolectomia esquerda com anastomose transversoretal; PCT-IT: Proctocolectomia total com ileostomia terminal; AAPCT: Amputação abdômino-perineal com colostomia definitiva; $R L$ : Ressecção local.

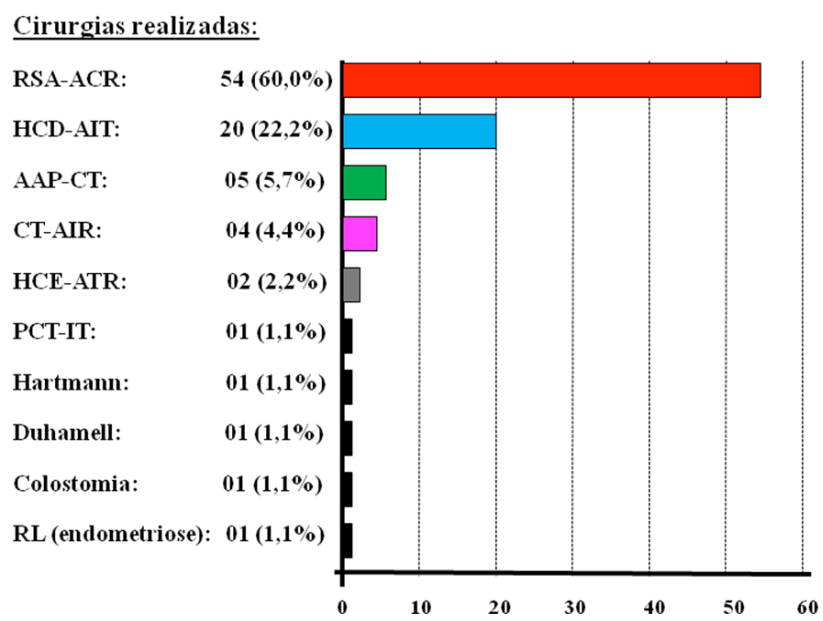

Siglas: RSA-ACR: Retossigmoidectomia abdominal com anastomose colorretal; HCD-AIT: Hemicolectomia direita com anastomose íleotransverso; CT-AIR: Colectomia total com anastomose ileo-retal; HCE-ATR: Hemicolectomia esquerda com anastomose transversoretal; PCT-IT: Proctocolectomia total com ileostomia terminal; AAPCT: Amputação abdomino-perineal com colostomia definitiva; $R L$ : Ressecção local.

Figura 6. Distribuição, por cirurgias realizadas, de 90 pacientes submetidos a cirurgias colorretais por via videolaparoscópica: a primeira coluna mostra a elevada incidencia de retossigmoidectomias abdominais com anastomose colorretal (54 casos; 60,0\%) $(p<0,05)$, seguidas, de longe, pelas hemicolectomias direitas com anastomose ileo-transverso (20 casos; 22,2\%). 


\section{Tempos gastos na cirurgia}

Os tempos de cirurgia (em minutos) foram os seguintes: até 60 minutos um caso $(1,1 \%)$, de 61 a 120 minutos seis casos $(6,6 \%)$, de 121 a 180 minutos 34 casos $(37,8 \%)$, de 181 a 240 minutos 24 casos $(26,7 \%)$, de 241 a 300 minutos 16 casos $(17,8 \%)$, de 301 a 360 minutos seis casos $(6,6 \%)$, de 361 a 420 minutos dois casos $(2,3 \%)$, de 421 a 480 minutos nenhum caso $(0 \%)$ e um caso em 540 minutos $(1,1 \%)$ (Tabela 7 e Figura 7). O tempo variou de 60 minutos (mínimo) a 540 minutos (máximo), com tempo médio de 203 minutos (3 horas e 23 minutos), ficando 58 casos $(64,5 \%)$ entre 120 e 240 minutos (duas e quatro horas) e 74 casos $(82,3 \%)$ entre 120 e 300 minutos (duas e cinco horas) (Figura 7).

\section{Anastomoses}

Não houve anastomoses em nove casos $(10,0 \%)$ - sete casos de colostomias $(7,8 \%)$, um de ileostomia $(1,1 \%)$ e uma ressecção local de endometriose $(1,1 \%)$.

\section{Tipos de anastomoses e suturas}

Das 81 anastomoses $(90,0 \%), 50$ foram intra-abdominais mecânicas $(55,6 \%)$ e seis foram extra-abdominais mecânicas $(6,6 \%)$, totalizando 56 anastomoses mecânicas $(62,2 \%) ; 25$ foram extra-abdominais manuais $(27,8 \%)$ (Tabela 8 e Figura 8$)$.

\section{Dissecção, hemostasia e secção}

Foram usados o eletrocautério em 68 pacientes (75,6\%), o Ultracision ${ }^{\circledR}$ em 19 pacientes $(21,1 \%)$ e o Ligasure ${ }^{\circledR}$ em três pacientes (3,3\%) (Tabela 9 e Figura 9).

\section{Grampeadores}

Foram usados grampeadores (para anastomoses e para fechamento de extremidades) em 75 pacientes $(83,4 \%)$, tendo sido o mais usado o conjunto "grampeador circular e grampeador linear articulado" (50 casos; $55,6 \%$ ), seguido pelo grampeador linear cortante (12 casos; $13,3 \%$ ), grampeador linear articulado (oito casos; $8,9 \%$ ) e grampeador circular (cinco casos; $5,6 \%$ ) (Tabela 10 e Figura 10$)$.

\section{Tipos de incisões abdominais}

Houve 70 incisões abdominais para extração de peças e confecções de anastomoses $(77,8 \%)$, sendo a mais comum a de Mallard (39 casos; $43,4 \%$ ), seguida pela mediana (22 casos; $24,4 \%$ ) e pela Pfannenstiel modificada (nove casos; $10,0 \%$ ); nos restantes 20 pacientes $(22,2 \%)$ não houve tais incisões por terem sido as cirurgias convertidas em 13 casos $(14,4 \%)$ e com colostomia (seis casos; $6,7 \%$ ) e ileostomia (um caso; 1,1\%) (Tabela 11 e Figura 11).

\section{Extensões das incisões abdominais}

As extensões das incisões abdominais nos 70 pacientes tiveram suas dimensões de $5 \mathrm{~cm}$ em cinco pacientes $(7,1 \%)$, entre 6 e $10 \mathrm{~cm}$ em 55 pacientes (78,6\%), e entre 11 e $15 \mathrm{~cm}$ em dez pacientes $(14,3 \%)$ (Tabela 12 e Figura 12). As incisões variaram entre 5 (mínima) e $15 \mathrm{~cm}$ (máxima), com média de 7,2 cm.

\section{Intercorrências peroperatórias e conversões cirúrgicas}

Houve 12 intercorrências que levaram a conversões cirúrgicas para laparotomia $(13,2 \%)$ : instrumentos inadequados e defeituosos - quatro casos $(4,4 \%)$, lesão da artéria ileocólica - dois casos $(2,2 \%)$, dificuldade de dissecção do mesorreto - dois casos $(2,2 \%)$, aderência tumoral à parede abdominal - um caso $(1,1 \%)$, dificuldade de identificação dos vasos mesentéricos - um caso $(1,1 \%)$, dificuldade de remoção da peça já liberada - um caso $(1,1 \%)$ e obesidade do paciente - um caso $(1,1 \%)$ (Tabela 13 e Figura 13).

\section{Dimensões de peças cirúrgicas}

As dimensões das peças cirúrgicas ficaram entre 15 e $20 \mathrm{~cm}$, com média de $18,4 \mathrm{~cm}$ em 19 pacientes $(21,1 \%)$, entre 21 e $30 \mathrm{~cm}$ em 51 pacientes, com média de $26,4 \mathrm{~cm}$ em 51 pacientes $(56,8 \%)$, entre 31 e 40 $\mathrm{cm}$, com média de $35,5 \mathrm{~cm}$ em sete pacientes $(7,8 \%)$, entre 41 e $50 \mathrm{~cm}$, com média de $46,4 \mathrm{~cm}$ em três pacientes $(3,3 \%)$, entre 51 e $60 \mathrm{~cm}$, com média de 55,3 $\mathrm{cm}$ em três pacientes $(3,3 \%)$, entre 61 e $70 \mathrm{~cm}$, média de 79,9 cm em um paciente $(1,1 \%)$, entre 71 e $100 \mathrm{~cm}$ em nenhum paciente $(0 \%)$, entre 101 e 110 , com média de $110 \mathrm{~cm}$ em um paciente $(1,1 \%)$, entre 111 e 120 $\mathrm{cm}$, com média de $130 \mathrm{~cm}$ em dois pacientes $(2,2 \%)$, entre 121 e $130 \mathrm{~cm}$, com média de $130 \mathrm{~cm}$ em um paciente $(2,2 \%)$, e sem peça em dois pacientes $(2,2 \%)$ (Tabela 14 e Figura 14). 
Rev bras Coloproct Cirurgia colorretal videolaparoscópica: experiência inicial na abordagem de 90 pacientes, no Programa de Pós-graduação

Tabela 7. Distribuição, por tempo de cirurgia (em minutos), de 90 pacientes submetidos a cirurgias colorretais por via videolaparoscópica: o tempo médio para realização das cirurgias foi de 203 minutos (3 horas e 23 minutos), com a grande maioria entre duas e três horas (34 casos; $37,8 \%$ ), seguidas pelas cirurgias realizadas entre três e quatro horas (24 casos; 26,7\%), a grande maioria delas (58 casos; $64,5 \%)$ entre duas e quatro horas $(p<0,05)$.

\begin{tabular}{lcc}
\hline Tempo de cirurgia (minutos) & $\mathbf{N}$ & $\mathbf{\%}$ \\
\hline Até 60 & 01 & 1,1 \\
$61-120$ & 06 & 6,6 \\
$121-180$ & 34 & 37,8 \\
$181-240$ & 24 & 26,7 \\
$241-300$ & 16 & 17,8 \\
$301-360$ & 06 & 6,6 \\
$361-420$ & 02 & 2,3 \\
$421-480$ & 00 & 0 \\
$481-540$ & 01 & 1,1 \\
TOTAL & 90 & 100,0 \\
\hline
\end{tabular}

Observações:

Tempo mínimo: $060 \mathrm{~m}(1 \mathrm{~h})-1$ (RSA)

Tempo máximo: $540 \mathrm{~m}(9 \mathrm{~h})-1$ (RSA)

Tempo médio: $203 \mathrm{~m}(3 \mathrm{~h} 23 \mathrm{~m})$

58; $64,5 \%: \quad 2 h-4 h$

$74 ; 82,3 \%: \quad 2 h-5 h$

$\underline{\text { Tempo de cirurgia (minutos): }}$

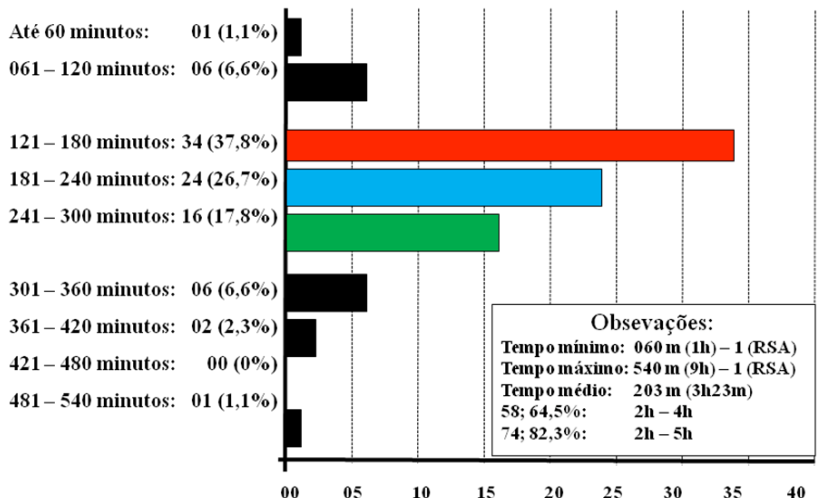

Figura 7. Distribuição, por tempo de cirurgia (em minutos), de 90 pacientes submetidos a cirurgias colorretais por via videolaparoscópica: o gráfico mostra a elevada incidência de cirurgias realizadas entre duas e três horas (34 casos; 37,8\%), seguidas pelas cirurgias realizadas entre três e quatro horas (24 casos; 26,7\%), a grande maioria delas (58 casos; 64,5\%) entre duas e quatro horas $(p<0,05)$.
Tabela 8. Distribuição, por tipo de anastomose, de 90 pacientes submetidos a cirurgias colorretais por via videolaparoscópica: a maioria marcante das cirurgias envolveu anastomoses intestinais (81 casos; 90,0\%), chamando a atenção à elevada incidência de anastomoses realizadas dentro da própria cavidade abdominal (50 casos; 55,6\%) $(p<0,05)$.

\begin{tabular}{lcr}
\hline Tipos de anastomose & N & \multicolumn{1}{c}{$\%$} \\
\hline Mecânica intra-abdominal & 50 & 55,6 \\
Manual extra-abdominal & 25 & 27,8 \\
Mecânica extra-abdominal & 06 & 6,6 \\
Total de anastomoses & $\mathbf{8 1}$ & $\mathbf{9 0 , 0}$ \\
Colostomias & 07 & 7,8 \\
Ileostomias & 01 & 1,1 \\
Ressecções locais & 01 & 1,1 \\
Total sem anastomose & $\mathbf{0 9}$ & $\mathbf{1 0 , 0}$ \\
Total & $\mathbf{9 0}$ & $\mathbf{1 0 0 , 0}$ \\
\hline
\end{tabular}

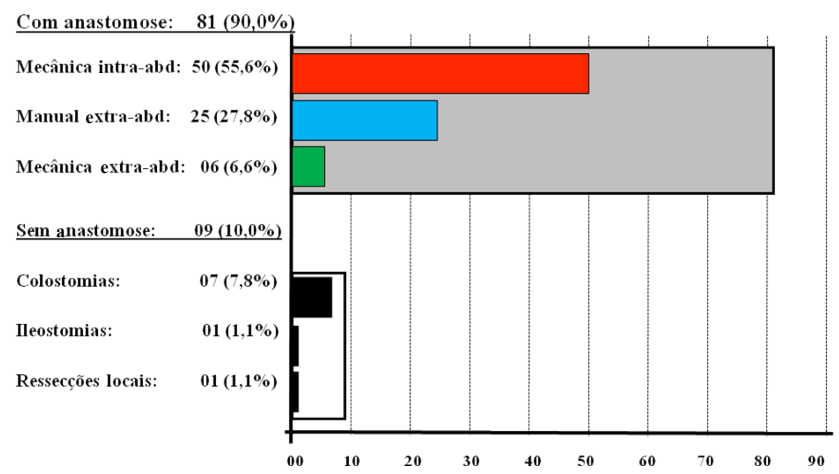

Figura 8. Distribuição, por tipo de anastomose, de 90 pacientes submetidos a cirurgias colorretais por via videolaparoscópica: a primeira coluna evidencia que a maioria marcante das cirurgias envolveu anastomoses intestinais (81 casos; 90,0\%), chamando a atenção à elevada incidência de anastomoses realizadas dentro da própria cavidade abdominal (50 casos; 55,6\%) $(p<0,05)$.

Tabela 9. Distribuição, por forma de dissecção $e$ hemostasia, de 90 pacientes submetidos a cirurgias colorretaisporviavideolaparoscópica: oeletrocautério foi o instrumento mais usado para dissecção, corte e hemostasia (68 casos; 75,6\%) $(p<0,05)$.

\begin{tabular}{lrr}
\hline Dissecção e hemostasia & N & \multicolumn{1}{c}{$\%$} \\
\hline Eletrocautério & 68 & 75,6 \\
Ultracision & 19 & 21,1 \\
Ligasure & 3 & 3,3 \\
Total & $\mathbf{9 0}$ & $\mathbf{1 0 0 , 0}$ \\
\hline
\end{tabular}




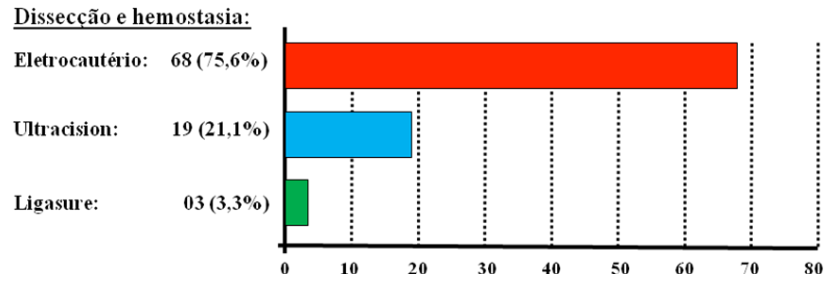

Figura 9. Distribuição, por forma de dissecção e hemostasia, de 90 pacientes submetidos a cirurgias colorretais por via videolaparoscópica: a primeira coluna mostra como o eletrocautério foi o instrumento mais usado para dissecção, corte e hemostasia (68 casos; 75,6\%) $(p<0,05)$.

Tabela 10. Distribuição, por uso de grampeadores, de 90 pacientes submetidos a cirurgias colorretais por via videolaparoscópica: a elevada incidência de anastomoses intra-abdominais justifica a semelhança com os tipos de grampeadores usados - associação de circulares com lineares articulados - (50 casos; $55,6 \%$ ).

\begin{tabular}{lrr}
\hline Grampeadores & N & \multicolumn{1}{c}{$\%$} \\
\hline Circular + Linear articulado & 50 & 55,6 \\
Linear cortante & 12 & 13,3 \\
Circular & 5 & 5,6 \\
Linear articulado & 8 & 8,9 \\
Pacientes com uso de grampeadores & 75 & 83,4 \\
Pacientes sem uso de grampeadores & 15 & 16,6 \\
& & \\
Sem anastomose & 09 & \\
Anastomoses manuais & 25 & \\
\hline
\end{tabular}

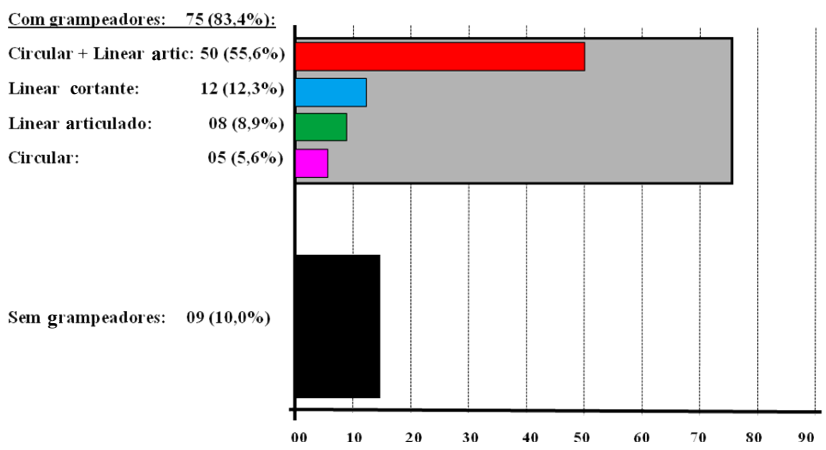

Figura 10. Distribuição, por uso de grampeadores, de 90 pacientes submetidos a cirurgias colorretais por via videolaparoscópica: a primeira coluna ressalta a grande incidência de uso da associação de circulares com lineares articulados (50 casos; $55,6 \%$ ).
Tabela 11. Distribuição, por incisão na parede abdominal, de 90 pacientes submetidos a cirurgias colorretais por via videolaparoscópica: houve incisões abdominais na grande maioria dos pacientes (70 casos; 77,8\%) $(p<0,05)$, sendo a incisão abdominal de Mallard a mais utilizada para anastomoses e remoção de peças cirúrgicas (39 casos; $43,4 \%)(p<0,05)$, seguida pela incisão mediana, com pouco mais da metade de casos (22 casos; $24,4 \%$ ).

\begin{tabular}{lcr}
\hline Incisões na parede abdominal & $\mathbf{N}$ & $\mathbf{\%}$ \\
\hline Mallard & 39 & 43,4 \\
Mediana & 22 & 24,4 \\
Pfannestiel & 09 & 10,0 \\
Total & 70 & 77,8 \\
Conversões & 13 & 14,4 \\
Colostomias & 06 & 6,7 \\
Ileostomias & 01 & 1,1 \\
Total & $\mathbf{2 0}$ & $\mathbf{2 2 , 2}$ \\
\hline
\end{tabular}

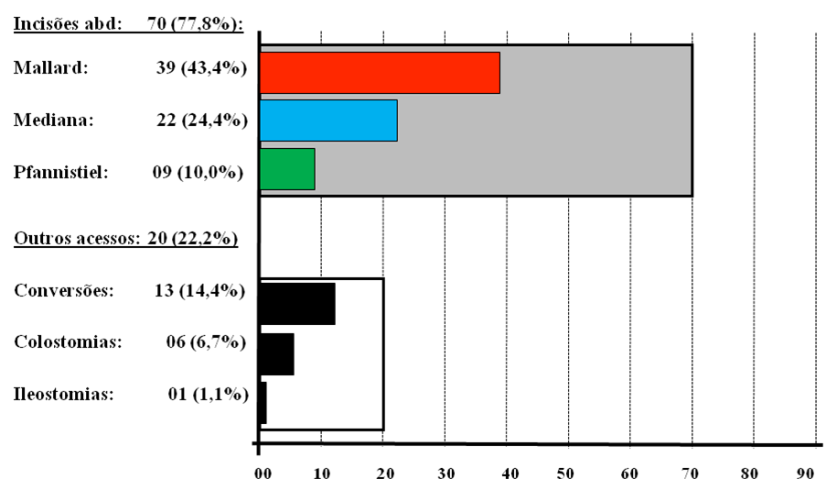

Figura 11. Distribuição, por incisão na parede abdominal, de 90 pacientes submetidos a cirurgias colorretais por via videolaparoscópica: a primeira coluna mais larga mostra a elevada incidência de incisões abdominais na grande maioria dos pacientes (70 casos; 77,8\%) $(p<0,05)$, sendo a incisão abdominal de Mallard a mais utilizada para anastomoses e remoção de peças cirúrgicas (39 casos; 43,4\%) $(p<0,05)$, mostrada pela primeira coluna estreita.

Tabela 12. Distribuição, por extensão das incisões abdominais, de 90 pacientes submetidos a cirurgias colorretais por via videolaparoscópica: as extensões mais comuns das incisões abominais ficaram entre 6 e $10 \mathrm{~cm}(55$ casos; 78,6\%) $(p<0,05)$, sendo a incisão menos extensa de $5 \mathrm{~cm}$ e a mais extensa de $15 \mathrm{~cm}$,com média de 7,2 cm.

\begin{tabular}{lcc}
\hline Extensão das incisões abdominais & N & $\mathbf{\%}$ \\
\hline Até $5 \mathrm{~cm}$ & 05 & 7,1 \\
$06-10 \mathrm{~cm}$ & 55 & 78,6 \\
$11-15 \mathrm{~cm}$ & 10 & 14,3 \\
Total & $\mathbf{7 0}$ & $\mathbf{1 0 0 , 0}$ \\
\hline
\end{tabular}

Incisão mais extensa: $15 \mathrm{~cm}$; incisão menos extensa: $5 \mathrm{~cm}$; extensão média: $7,2 \mathrm{~cm}$. 
Rev bras Coloproct Abril/Junho, 2011

\section{Cirurgia colo} sensu lato (residência e especialização) em Coloproctologia, pelo Grupo de Coloproctologia de Belo Horizonte
Vol. 31 Antonio Carlos Barros Lima Junior e Cols.

Extensão das incisões

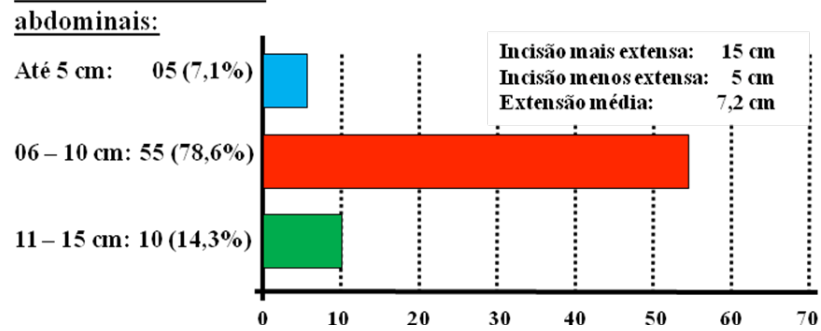

Figura 12. Distribuição, por extensão das incisões abdominais, de 90 pacientes submetidos a cirurgias colorretais porvia videolaparoscópica: as extensões mais comuns das incisões abominais ficaram entre 6 e $10 \mathrm{~cm}$ (55 casos; 78,6\%) $(p<0,05)$, como demonstrado pela segunda coluna, sendo a incisão menos extensa de $5 \mathrm{~cm}$ e a mais extensa de 15 $\mathrm{cm}$, com média de $7,2 \mathrm{~cm}$.

Tabela 13. Distribuição, por intercorrências que levaram a conversões para laparotomia, de 90 pacientes submetidos a cirurgias colorretais por via videolaparoscópica: 12 intercorrência levara à conversão cirúrgica $(13,2 \%)$, sendo a causa mais frequente os instrumentos inadequados e defeituosos (quatro casos; 4,4\%) ( $p>0,05)$.

\begin{tabular}{lrc}
\hline $\begin{array}{l}\text { Intercorrências que levaram a } \\
\text { conversões para laparotomia }\end{array}$ & $\mathbf{N}$ & $\mathbf{\%}$ \\
\hline Instrumentos inadequados e defeituosos & 4 & 4,4 \\
Lesão de artéria ileocólica & 2 & 2,2 \\
Dificuldade dissecção de mesorreto & 2 & 2,2 \\
Aderência tumoral ao peritônio & 1 & 1,1 \\
Identificação de vasos mesentéricos & 1 & 1,1 \\
Dificuldade para remoção da peça & 1 & 1,1 \\
Obesidade & 1 & 1,1 \\
Total & $\mathbf{1 2}$ & $\mathbf{1 3 , 2}$ \\
\hline
\end{tabular}

Dimensões de peças cirúrgicas $(\mathrm{cm})$ :

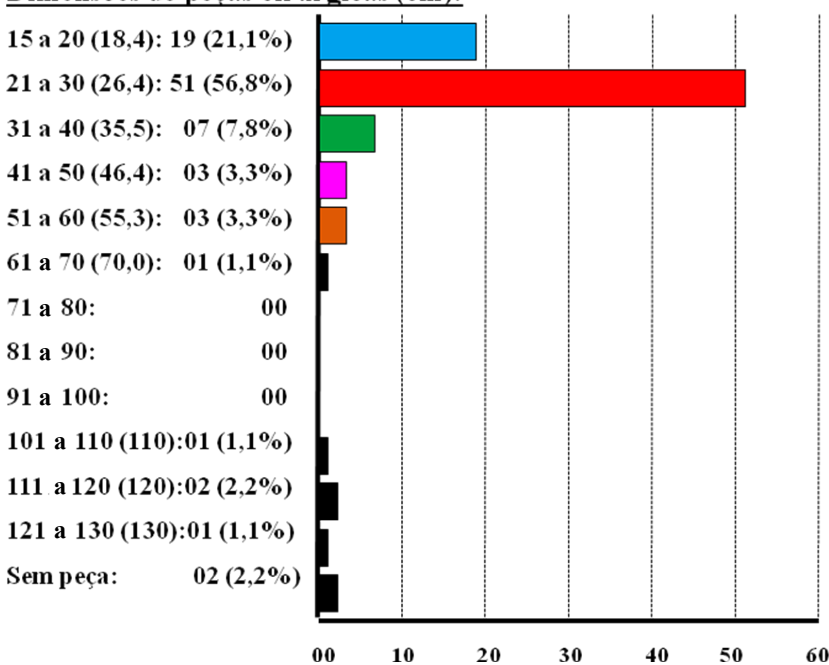

Figura 13. Distribuição, por dimensões de peças cirúrgicas, de 90 pacientes submetidos a cirurgias colorretaisporvia videolaparoscópica: a segunda coluna, representativa das dimensões (medindo entre 21 e $30 \mathrm{~cm}$, com média de 26,4 cm), com 51 casos $(56,8 \%)(p<0,05)$, demonstra que foram essas as dimensões mais comuns.
Tabela 14. Distribuição, por dimensões de peças cirúrgicas, de 90 pacientes submetidos a cirurgias colorretais por via videolaparoscópica: a grande maioria das peças cirúrgicas mediam entre 21 e $30 \mathrm{~cm}$, com média de 26,4 cm (51 casos; 56,8\%) $(p<0,05)$, com média universal de $33,2 \mathrm{~cm}$.

\begin{tabular}{lccc}
\hline Dimensões de peças cirúrgicas & N & Média & \% \\
\hline 15 a $20 \mathrm{~cm}$ & 19 & 18,4 & 21,1 \\
21 a $30 \mathrm{~cm}$ & 51 & 26,4 & 56,8 \\
31 a $40 \mathrm{~cm}$ & 07 & 35,5 & 7,8 \\
41 a $50 \mathrm{~cm}$ & 03 & 46,4 & 3,3 \\
51 a $60 \mathrm{~cm}$ & 03 & 55,3 & 3,3 \\
61 a $70 \mathrm{~cm}$ & 01 & 70,0 & 1,1 \\
71 a $80 \mathrm{~cm}$ & 00 & 00 & 0 \\
81 a $90 \mathrm{~cm}$ & 00 & 00 & 0 \\
91 a $100 \mathrm{~cm}$ & 00 & 00 & 0 \\
$101-110 \mathrm{~cm}$ & 01 & 110 & 1,1 \\
$111-120 \mathrm{~cm}$ & 02 & 120 & 2,2 \\
121 a $130 \mathrm{~cm}$ & 01 & 130 & 1,1 \\
Sem peça & 02 & 00 & 2,2 \\
Total & $\mathbf{9 0}$ & $\mathbf{3 3 , 2} \mathbf{~ c m}$ & $\mathbf{1 0 0 , 0}$ \\
\hline
\end{tabular}

Permanência hospitalar (dias):

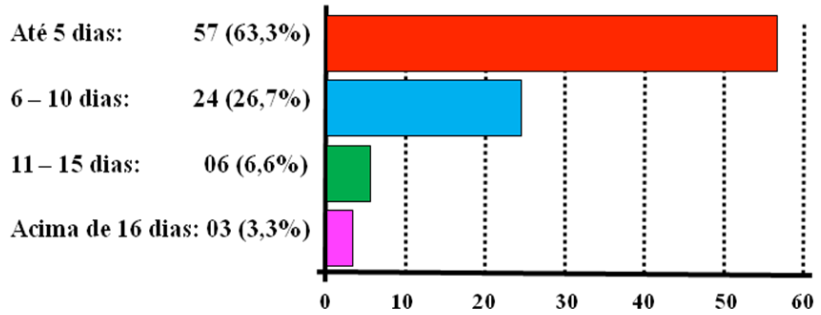

Figura 14. Distribuição, por permanência hospitalar, de 90 pacientes submetidos a cirurgias colorretais por via videolaparoscópica: a primeira coluna (internamento abaixo de cinco dias) demonstra ter sido esta a permanência mais comum (57 pacientes; 63,3\%) ( $p<0,05)$, com média de 5,3 dias.

\section{Morbimortalidade}

Houve 13 complicações pós-operatórias (14,4\%), sendo 11 cirúrgicas $(12,2 \%)$, representadas por deiscências de anastomose em quatro pacientes (4,5\%), infecção de porte em dois pacientes $(2,2 \%)$, hérnia incisional em um paciente $(1,1 \%)$, abscesso e hemorragia em um paciente $(1,1 \%)$, fístula retovaginal em um paciente $(1,1 \%)$, necrose de colostomia em um paciente $(1,1 \%)$ e enterorragia em um paciente $(1,1 \%)$. Resultaram dois óbitos cirúrgicos $(2,2 \%)$ de dois ca- 
sos de deiscência de anastomose. As complicações clínicas foram insuficiência cardíaca em um paciente $(1,1 \%)$ e caquexia com senectude (paciente com 93 anos), este último com êxito letal por falência múltipla de órgãos (Tabela 15).

\section{Permanência hospitalar}

Ficaram internados menos de cinco dias 57 pacientes $(63,3 \%)$, entre 6 e 10 dias 24 pacientes $(26,7 \%)$, entre 11 e 15 dias seis pacientes (6,7\%), e acima de 16 dias, em decorrência de complicações e intercorrências clínicas, três pacientes $(3,3 \%)$, dos quais, um por 28 , outro por 39 e outro por 40 dias. A permanência variou de 3 (seis pacientes) a 15 dias, com média de 5,3 dias (excluídos os três casos salientados) (Tabela 16 e Figura 15).

\section{Internação pós-operatória em CTI}

Dos 90 pacientes, 62 não se internaram no CTI $(68,9 \%)$, sendo que, dos 28 internados $(31,1 \%), 12$ permaneceram por apenas um dia $(13,4 \%)$, nove por dois dias $(10,0 \%)$, dois por três dias $(2,2 \%)$, dois por quatro dias $(2,2 \%)$, um por seis dias $(1,1 \%)$ e dois por longo tempo estendido até o óbito (Tabela 17).

\section{Comorbidades}

Dos 90 pacientes, 22 apresentaram comorbidades (24,4\%): 11 casos de hipertensão arterial sistêmica exclusiva $(12,2 \%)$, três casos de cardiopatia coronariana dos quais dois com AVC prévio (3,3\%), três casos de diabete mélito com hipertensão arterial sistêmica $(3,3 \%)$, dois casos de obesidade com dislipidemia e hipertensão $(2,2 \%)$, um caso de subobstrução intestinal em retocolite ulcerativa grave $(1,1 \%)$, um caso de cardiopatia chagásica grave $(1,1 \%)$ e um caso de senectude de 93 anos com arteriosclerose marcante $(1,1 \%)$ (Tabela 18$)$.

\section{Liberação de dieta oral}

A dieta oral foi iniciada com 24 horas em 49 pacientes $(54,5 \%)$, em dois dias em 32 pacientes $(35,6 \%)$, em três dias em quatro pacientes $(4,4 \%)$, em quatro dias em dois pacientes $(2,2 \%)$, ficando sem liberação em três pacientes, que vieram a falecer no CTI (3,3\%) (Tabela 19).

\section{Sumário dos resultados}

Foram os seguintes os resultados observados, resumidamente:
1. Média etária: 62 anos ( 25 a 93 anos).

2. Gênero: feminino (52 pacientes; $57,8 \%$ ).

3. Preparo intestinal mais realizado: Picolax (53 casos; $58,9 \%$ ).

4. Colonoscopia com biópsia: 76 pacientes $(84,4 \%)$.

5. Diagnóstico principal: câncer colorretal (60 casos; $66,7 \%)$.

6. Cirurgias mais realizadas: retossigmoidectomias (54 casos; 60,0\%).

7. Tempo de cirurgia: entre duas e três horas (34 casos, 37,8\%); média de 203 minutos.

8. Anastomoses: 81 casos $(90,0 \%)$, a maioria mecânica intra-abdominal $(55,6 \%)$.

9. Grampeadores mais usados: circulares com lineares articulados (50 casos; 55,6\%).

10. Eletrocautério foi usado em 68 pacientes $(75,6 \%)$.

11. Incisão abdominal mais usada: Mallard (39 casos; 43,4\%).

12. Extensões mais comuns das incisões: 6 a 10 cm (55 casos; $78,6 \%$ ).

13. Intercorrências peroperatórias com conversões: 12 $(13,2 \%)$.

14. Dimensão média das peças cirúrgicas: $33,2 \mathrm{~cm}$.

15. Complicações pós-operatórias: 13 (14,4\%), sendo 11 cirúrgicas $(12,2 \%)$.

16. Óbitos: três (dois clínicos e um cirúrgico).

17. Tempo médio de internamento: 5,3 dias, sendo 57 $(63,3 \%)$ a até cinco dias.

18. Internamento em CTI: 28 pacientes $(31,1 \%)$, mais por comorbidades (22 casos; 24,4\%).

19. Liberação de dieta oral: um dia para 49 pacientes $(54,5 \%)$.

\section{Documentação de casos de cirurgias colorretais videolaparoscópicas}

A Figura 15 mostra uma retossigmoidectomia abdominal com anastomose colorretal para câncer no sigmoide por videolaparoscopia - peça cirúrgica fechada (A) e aberta (B), e portes e incisão abdominais para remoção da peça cirúrgica (C). A Figura 16 mostra uma hemicolectomia direita com anastomose íleo-transverso para câncer no ceco por videolaparoscopia - peça cirúrgica fechada $(\mathrm{A})$ e portes e incisão abdominais para remoção da peça cirúrgica (B). A Figura 17 é de uma proctocolectomia total com ileostomia em alça para doença de Crohn ileocecal e anoperineal por videolaparoscopia - inspecção do períneo do paciente (A), térmi- 
Rev bras Coloproct Cirurgia colorretal videolaparoscópica: experiência inicial na abordagem de 90 pacientes, no Programa de Pós-graduação

Tabela 15. Distribuição, por morbimortalidade pósoperatória, de 90 pacientes submetidos a cirurgias colorretais por via videolaparoscópica: 13 foi o número total de complicações (14,4\%), mas 11 foram as decorrentes do ato cirúrgico (12,2\%), salientando a deiscência de anatomose (quatro casos; 4,5\%) ( $p>0,05)$; houve dois óbitos cirúrgicos e um clínico.

\begin{tabular}{|c|c|c|}
\hline Complicações pós-operatórias & $\mathbf{N}$ & $\%$ \\
\hline Complicações cirúrgicas & 11 & 12,2 \\
\hline Deiscência anastomose* & 4 & 4,5 \\
\hline Hérnia incisional & 1 & 1,1 \\
\hline Infecção de porte & 2 & 2,2 \\
\hline Abscesso e hemorragia & 1 & 1,1 \\
\hline Fístula retovaginal & 1 & 1,1 \\
\hline Necrose de colostomia & 1 & 1,1 \\
\hline Enterorragia & 1 & 1,1 \\
\hline Complicações clínicas & 2 & 2,2 \\
\hline Insuficiência cardíaca & 1 & 1,1 \\
\hline Estado geral (93 anos) $)^{* *}$ & 1 & 1,1 \\
\hline Total & 13 & 14,4 \\
\hline
\end{tabular}

(*) Óbitos cirúrgicos: dois. (**) Óbito clínico: um.
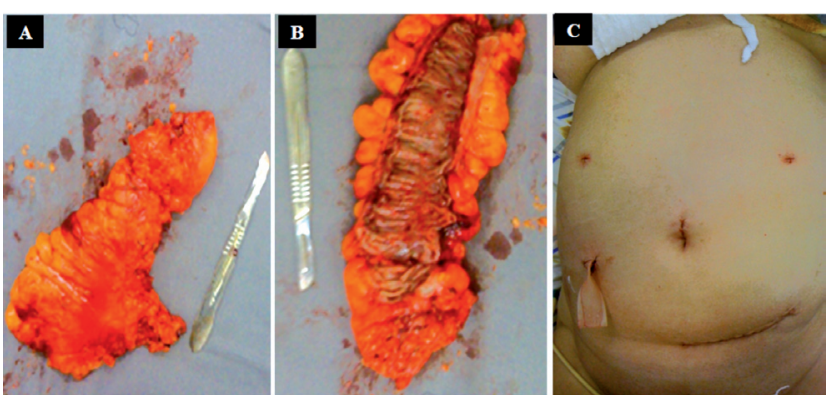

Figura 15. Retossigmoidectomia com anastomose colorretal para câncer no sigmoide por videolaparoscopia: peça cirúrgica fechada (A) e aberta (B) e portes e incisão abdominais para remoção da peça cirúrgica (C).

Tabela 16. Distribuição, por permanência hospitalar, de 90 pacientes submetidos a cirurgias colorretais por via videolaparoscópica: a maioria dos pacientes operados permaneceu no hospital menos de cinco dias (57 pacientes; 63,3\%) ( $p<0,05)$, com média de 5,3 dias.

\begin{tabular}{lcr}
\hline Permanência hospitalar (dias) & N & \% \\
\hline Até 5 dias & 57 & 63,3 \\
$6-10$ dias & 24 & 26,7 \\
$11-15$ dias & 06 & 6,7 \\
16 dias ou mais $(40,39,28)$ & 03 & 3,3 \\
Total & 90 & 100,0 \\
\hline
\end{tabular}

Permanência mais curta: três dias (seis pacientes).

Permanência mais longa: 40, 39, 28 dias (três pacientes).

Permanência média: 5,3 dias.

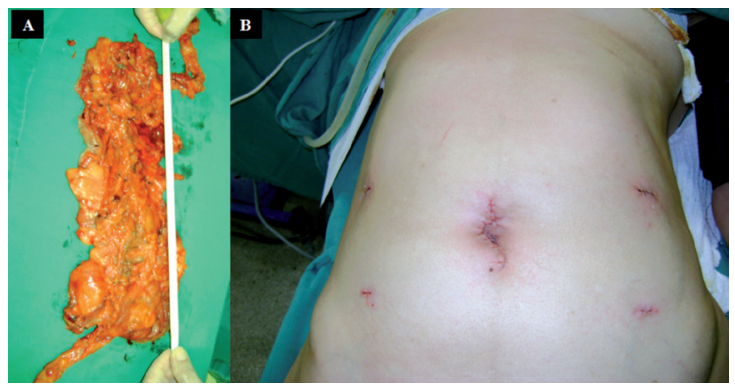

Figura 16. Hemicolectomia direita com anastomose íleo-transverso para câncer no ceco por videolaparoscopia: peça cirúrgica fechada (A) e portes e incisão abdominais para remoção da peça cirúrgica (B).

Tabela 17. Distribuição, por permanência em CTI, de 90 pacientes submetidos a cirurgias colorretais por via videolaparoscópica: 62 pacientes não precisaram de internamento em CTI $(68,9 \%)(p<0,05)$, sendo que dos que se internaram a permanência mais comum foi de um dia (12 pacientes; $13,4 \%)(p>0,05)$.

\begin{tabular}{lcc}
\hline CTI (dias) & N & $\mathbf{0}$ \\
\hline Sem CTI & 62 & 68,9 \\
Com CTI & 28 & 31,1 \\
1 dia & 12 & 13,4 \\
2 dias & 09 & 10,0 \\
3 dias & 02 & 2,2 \\
4 dias & 02 & 2,2 \\
5 dias & 00 & 0 \\
6 dias & 01 & 1,1 \\
Longo tempo (óbito) & 02 & 2,2 \\
Total & 90 & 100,0 \\
\hline
\end{tabular}

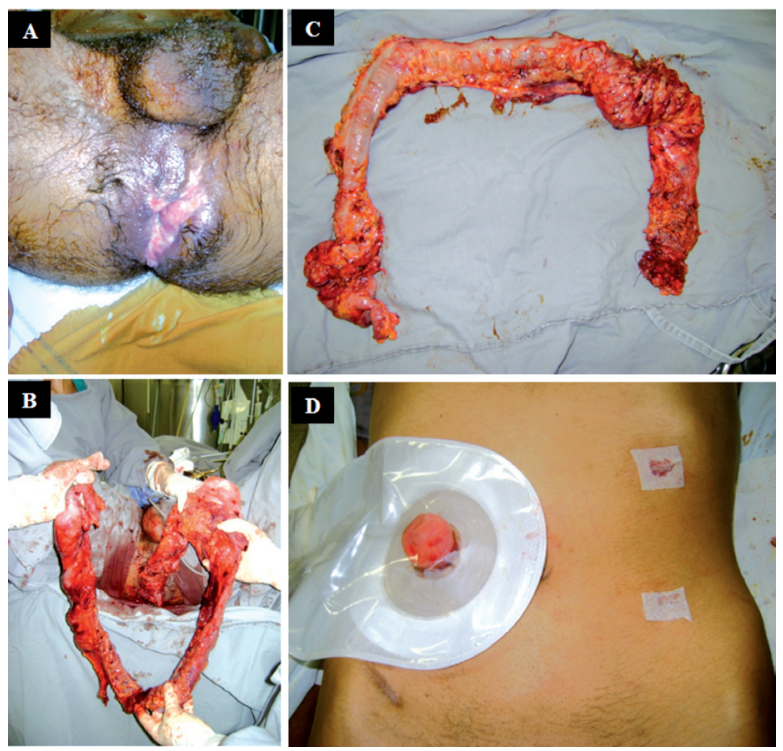

Figura 17. Proctocolectomia total com ileostomia em alça para doença de Crohn ileocecal e anoperineal por videolaparoscopia: inspecção do períneo do paciente (A), término da ciúrgia (B), vista panorâmica da peça cirúrgica fechada $(C)$ e portes e incisão abdominais para remoção da peça cirúrgica (D). 
Tabela 18. Distribuição, por comorbidades, de 90 pacientes submetidos a cirurgias colorretais por via videolaparoscópica: houve 22 pacientes com comorbidades $(24,4 \%)$, destacando-se a hipertensão arterial sistêmica (11 casos; 12,2\%).

\begin{tabular}{lrr}
\hline Comorbidades & N & $\mathbf{0} \%$ \\
\hline Hipertensão arterial sistêmica (HAS) & 11 & 12,2 \\
Cardiopatia e coronariopatia (1 AVC) & 3 & 3,3 \\
Diabetes mellitus (2 com HAS) & 3 & 3,3 \\
Subobstrução intestinal e RCUI & 1 & 1,1 \\
Obesidade (1 com dislipidemia) & 2 & 2,2 \\
Idade (93 anos) & 1 & 1,1 \\
Cardiopatia chagásica & 1 & 1,1 \\
Total & $\mathbf{2 2}$ & $\mathbf{2 4 , 4}$ \\
\hline
\end{tabular}

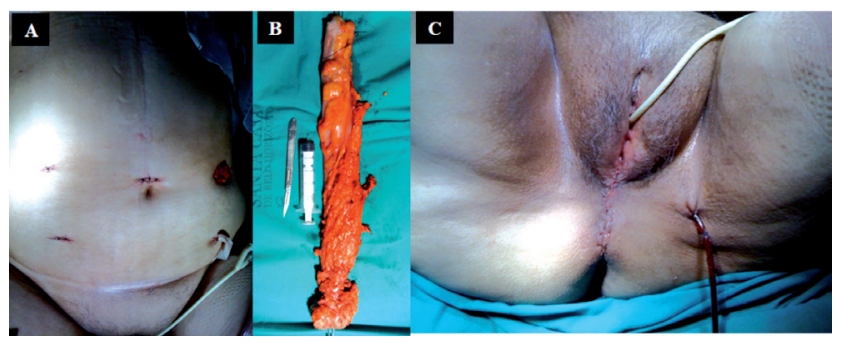

Figura 18. Amputação abdominoperineal com colostomia terminal para câncer retal baixo por videolaparoscopia: portes e estoma abdominais (A), vista panorâmica da peça cirúrgica fechada $(B)$ e aspecto final do períneo $(C)$.

Tabela 19. Distribuição, por dias de liberação de dieta oral, de 90 pacientes submetidos a cirurgias colorretais por via videolaparoscópica: a dieta oral foi liberada para 49 pacientes após 24 horas da cirurgia $(54,5 \%)(p<0,05)$.

\begin{tabular}{lcr}
\hline Dias & $\mathbf{N}$ & $\mathbf{0}$ \\
\hline 1 dia & 49 & 54,5 \\
2 dias & 32 & 35,6 \\
3 dias & 04 & 4,4 \\
4 dias & 02 & 2,2 \\
Sem liberação & 03 & 3,3 \\
Total & $\mathbf{9 0}$ & $\mathbf{1 0 0 , 0}$ \\
\hline
\end{tabular}

no da cirurgia (B), vista panorâmica da peça cirúrgica fechada $(C)$ e portes e incisão abdominais para remoção da peça cirúrgica (D). A Figura 17 é uma amputação abdominoperineal com colostomia terminal para câncer retal baixo por videolaparoscopia: portes e estoma abdominais (A), vista panorâmica da peça cirúrgica fechada (B) e aspecto final do períneo (C).

\section{DISCUSSÃO}

A aplicação do acesso laparoscópico para os procedimentos colorretais representa um grande avanço no sentido de proporcionar recuperação mais rápida dos pacientes. Os materiais utilizados nesses procedimentos e o tempo operatório estão associados a custos mais elevados. No entanto, o acúmulo de experiência do cirurgião associado à diminuição de tempo operatório, à tendência natural de redução de preço dos materiais à medida que surgem tecnologias mais avançadas e ao emprego de medidas de fast track demonstram potencial para contrabalançar e até mesmo superar os custos impostos pelo método, tornando viável e possivelmente vantajosa a via laparoscópica nos procedimentos colorretais ${ }^{1}$.

Em 1990, foram executadas as primeiras cirurgias laparoscópicas planejadas, cabendo a Moises Ja$\operatorname{cobs}^{2}$ a primeira colectomia direita assistida, a Dennis Fowler $^{3}$ a primeira colectomia esquerda assistida e a Morris Franklin o primeiro procedimento totalmente intracorpóreo. No Brasil, Regadas et al. ${ }^{4}$ realizaram a primeira intervenção laparoscópica para reconstrução do trânsito intestinal pós-operação de Hartmann em 1992.

As principais críticas com relação à videocirurgia dizem respeito à maior curva de aprendizado, maior tempo operatório, segurança e maior custo. Outra preocupação é a perda da sensação tátil durante o ato operatório, pois em procedimentos laparoscópicos essa sensação ainda é bastante limitada com os instrumentos atualmente disponíveis ${ }^{5,6}$.

Os Programas de Especialização-Residência em Coloproctologia estão em processo de adaptação para adequar seus currículos para a implementação do treinamento em cirurgia colorretal laparoscópica. Vivemos um momento em que os preceptores estão deixando a curva de aprendizado e muitos programas já estão treinando seus residentes. Em 2004, a ASCRS (The American Society of Colon and Rectal Surgeons) e a SAGES (The Society of American Gastrointestinal and Endoscopic Surgeons), visando ao treinamento de cirurgiões colorretais e gerais, desenvolveram o Focus Group on Laparoscopic Colectomy Education, um programa baseado, resumidamente, em cinco módulos: didático, laboratório em suínos, laboratório em simuladores, laboratório em cadáveres e prático (hands on ${ }^{7}$. 
Os primeiros 90 casos realizados dentro do Programa de Pós-graduação lato sensu em Coloproctologia pelo Grupo de Coloproctologia da Santa Casa de Belo Horizonte foram estudados e compilados nesse estudo.

Em relação aos dados demográficos, podemos observar que mais da metade dos pacientes eram do sexo feminino $(57,8 \%)$ e a faixa etária predominante, em 27 pacientes, foi de 51 a 60 anos. Dos 90 pacientes, 86 foram submetidos à colonoscopia para diagnóstico etiológico, sendo que em 76 foram obtidos espécimes para estudo histopatológico. Os demais pacientes foram submetidos à retossigmoidoscopia, com impossibilidade de ultrapassar lesões retais, ou tiveram seus diagnósticos por meio de exames de imagem. Foram diagnosticados 60 casos de câncer colorretal, sendo o reto e o sigmoide os sítios mais comuns, seguidos de 12 casos de pólipos colorretais, sete casos de doença diverticular e outros diagnósticos em 11 casos. Analisando os dados de trabalhos nacionais e internacionais, verificamos que o perfil das patologias colorretais abordadas por via laparoscópica vem se alterando. Até 2000, os grandes levantamentos mostravam as patologias benignas como as mais frequentes, ficando as neoplasias em segundo lugar. Campos et al. ${ }^{1}$, em estudo retrospectivo multicêntrico publicado em 2001, demonstraram que, de 1843 pacientes operados, 1109 eram portadores de doença colorretal benigna contra 734 portadores de tumores malignos. Em nossa série, o câncer colorretal foi a patologia predominante com significância estatística. Quanto às neoplasias colônicas, séries recentes demonstraram não haver alteração dos índices de recidiva e sobrevida. Entretanto, a avaliação dos resultados oncológicos nas ressecções retais ainda suscita controvérsias ${ }^{8}$.

As cirurgias mais realizadas foram as retossigmoidectomias $(60 \%)$ e a colectomia direita $(22,2 \%)$, e o intervalo de tempo cirúrgico gasto foi de 121 a 180 minutos em $37,8 \%$ dos casos. Regadas et al. ${ }^{4}$ tiveram como tempo médio 166,3 minutos nos seus 401 primeiros casos. Em nossa instituição, um hospital público, ainda temos dificuldade para aquisição de recursos tecnológicos, como as tesouras coaguladoras e os endogrampeadores; dos 90 procedimentos realizados, esses recursos foram utilizados em apenas 22 casos $(24,4 \%)$. Como alternativa de dissecção, utilizamos o eletrocautério em 75,6\% dos casos, o que tornou o procedimento mais trabalhoso e com tempo operatório mais prolongado. Talvez esses fatores justifiquem o fato de que nos 12 casos de conversão $(13,2 \%)$ os instrumentos inadequados fossem a causa mais comum de intercorrências, seguidos de lesões vasculares e aderências. As taxas de conversão na literatura variam entre $0 \%$ e $23 \%{ }^{1,8,9}$.

As incisões abdominais auxiliares representam alternativas táticas extremamente importantes na videocirurgia colorretal, principalmente em procedimentos híbridos. Durante procedimentos laparoscópicos, as incisões auxiliares podem ser realizadas com três objetivos, a saber: 1) retirar a peça cirúrgica; 2) facilitar determinado passo cirúrgico e aumentar a segurança do procedimento; 3) possibilitar a realização de anastomoses manuais e, assim, diminuir os custos do procedimento ${ }^{10}$. Na nossa casuística, foram realizadas 70 incisões, sendo a de Mallard em 39 casos (43,4\%), seguida da Pfannenstiel nas retossigmoidectomias e a mediana médio-umbilical nas colectomias direitas. No tocante a dimensões de peças cirúrgicas, 56,8 \% dos espécimes tinham média de $26,4 \mathrm{~cm}$. Esses dados terão maior interesse em outro trabalho da série que compara com espécimes obtidos por laparotomia.

A morbimortalidade pós-operatória foi dividida em dois subgrupos: complicações cirúrgicas e complicações clínicas. As complicações cirúrgicas foram observadas em 11 casos $(12,2 \%)$, representadas pela deiscência de anastomose $(4,5 \%)$, infecção de incisão auxiliar, abscesso intracavitário e hemorragia. As complicações clínicas representaram $2,2 \%$ da morbimortalidade. As complicações mais frequentes são representadas por lesão visceral (intestino delgado, grosso, bexiga ou ureter) devido à utilização de instrumental cirúrgico inadequado, manobras intempestivas ou dificuldade no reconhecimento anatômico quando da presença de aderências excessivas intraperitoniais ${ }^{9}$. Em relação ao tempo de permanência hospitalar, $63,3 \%$ dos pacientes ficaram menos de cinco dias internados, $68,9 \%$ dos pacientes não necessitaram de permanência no CTI pós-operatório e 54,4 \% receberam dieta oral líquida no primeiro dia de pósoperatório. Em grandes séries multicêntricas, se observa um período de internação hospitalar mais curto quando comparado com pacientes com característi- 
cas semelhantes submetidos à cirurgia laparotômica. A menor permanência hospitalar pode ser atribuída a menores índices de infecção, retorno mais precoce das funções intestinais e da dieta oral ${ }^{11}$. Braga et al. ${ }^{12}$ verificaram que a utilização da técnica laparoscópica reduz a taxa de complicações pós-operatórias, infecções e o tempo de permanência hospitalar. A maioria dos fatores de risco para morbidade pós-operatória identificada na cirurgia convencional desapareceu no grupo laparoscópico.

\section{CONCLUSÃO}

Os primeiros 90 casos permitem aos autores concluir ser a CVL uma técnica cirúrgica factível, segura e adequada para a abordagem das doenças dos cólons e do reto, ensejando resultados satisfatórios, que em nada ficam a dever às abordagens por cirurgia aberta.

A aferição feita com dados da literatura permite verificar que os resultados são condizentes com os da literatura correlata.

ABSTRACT: The objective was to undertake a careful review of a sample of 90 patients who underwent laparoscopic colorectal resections through the course of 12 months (May 2009 to May 2010). The average age was 62.1 years, with extremes of 20 and 93 years, mostly female (52 patients; 57.8\%). Bowel preparation was performed with Picolax in 53 patients (58.9\%); 76 patients underwent colonoscopy and biopsy (84.4\%). Colorectal cancer was the most common disease (60 cases; $66.7 \%)$, followed by polyps (12 cases; $13.4 \%$ ), diverticular disease (7 cases; $7.8 \%$ ) and other diseases (11 cases; $12,1 \%)$. The surgeries performed were retosigmoidectomy (54 cases; $60.0 \%$ ), followed by right hemicolectomy (20 cases; $22.2 \%$ ) and others. Most of the surgeries were carried out between 2 and 3 hours ( 34 cases; $37.8 \%$ ) and between 3 and 4 hours ( 24 cases; 26.7\%), with an average of 203 minutes. In 81 cases, there were anastomosis $(\mathbf{9 0 . 0} \%)$, most mechanical intra-abdominal $(\mathbf{5 5 . 6} \%)$ and manual extra-abdominal $(25$ cases, $27.8 \%)$, being the set of circular and linear articulated staplers the most commonly used features (50 cases, $55.6 \%)$. Electrocautery was used in 68 patients (75.6\%). The most used abdominal incision was Mallard incision (39 cases; $43.4 \%$ ) and median incision (22 cases; $24.4 \%$ ), being the most common extensions between 6.0 and $10.0 \mathrm{~cm}$ (55 cases; $78.6 \%$ ). There were 12 postoperative complications (13.2\%), leading to conversions to laparotomy. The average size of surgical specimens was $33.2 \mathrm{~cm}$, mostly between 21 and $30 \mathrm{~cm}(51 \mathrm{cases} ; 56.8 \%)$. There were 13 postoperative complications (14.4\%), being 11 surgical $(12.2 \%)$ and two clinics $(2.2 \%)$ of which took place three deaths, being two from surgery and one from comorbidities. The mean length of hospital stay was 5.3 days, and 57 patients (63.3\%) up to 5 days. 28 patients were admitted in the ICU (31.1\%), mainly because of comorbidities (22 cases; $24.4 \%)$. The release of oral intake was a day for 49 patients $(\mathbf{5 4 . 5} \%)$. The authors compare the results with related literature and confirm the similarities.

Keywords: colorectal surgery; laparoscopy.

\section{REFERÊNCIAS}

1. Campos FGCM, Souza Júnior AHS, Carmel APW, HabrGama A, Gonçalves CA, Cordeiro F, et al. Cirurgia laparoscópica colorretal. Resultados do Inquérito Nacional Brasileiro - 2001. Rev bras Coloproct 2001;21(3):13543.

2. Jacobs M, Verdeja JC, Gosdstein HS. Minimally invasive colon resection (laparoscopic colectomy). Surg Laparosc Endosc 1991;1:138-43.

3. Fowler DL, White SA. Laparoscopic assisted sigmoid resection. Surg Laparosc Endosc 1991;1:183-8.

4. Regadas FSP, Regadas SMM, Rodrigues LV, Lima D, Silva FR, Regadas Filho FSP. Cirurgia colorretal laparoscópica. Experiência com 401 casos. Rev bras videocir 2005;3(4):191-5.
5. Dinçler S, Koller MT, Steurer J, Bachmann LM, Christen D, Buchmann P. Multidimensional analysis of learning curvesin laparoscopic sigmoid resection: eight-year results. Dis Colon Rectum 2003;46:1371-9.

6. Campos FGCM, Caravatto PP, Araújo SEA. Avaliação dos efeitos imunológicos em operações colo-retais minimamente invasivas. Rev bras Coloproct 2005;25(3):285-92

7. Fleshman J, Marcello P, Stamos MJ, Wexner SD, Focus Group on Laparoscopic Colectomy Education as endorsed by The American Society of Colon and Rectal Surgeons (ASCRS) and The Society of American Gastrointestinal and Endoscopic Surgeons (SAGES) Dis Colon Rectum 2006;49:945-9.

8. Melani AGF, Campos FG. Ressecção laparoscópica pósterapia neo-adjuvante no tratamento do câncer no reto médio e baixo. Resultados da Fundação Pio XII - Hospital de Câncer de Barretos. Rev bras Coloproct 2006;26(1):89-96. 
9. Wu FPK, Hoekman K, Sietses C, von Blomberg BM, Meijer $\mathrm{S}$, Bonjer HJ, et al. Systemic and peritoneal angiogenic response after laparoscopic or conventional colon resection in cancer patients: a prospective, randomized trial. Dis Colon Rectum 2004;47:1670-4.

10 Lupinacci RA, Pedroso MA, Lupinacci RM, Farah FJM, Campos FGCM. Incisões auxiliares em cirurgia colo-retal vídeo-laparoscópica. Rev bras Coloproct 2005;25(2):192-8.

11. Bardram L, Funch-Jensen P, Kehlet H. Rapid rehabilitation in elderly patients after laparoscopic colonic resection. Br J Surg 2000;87:1540-5.
12. Braga M, Vignali A, Zuliani W, Frasson M, Radaelli G, Di Carlo V, Laparoscopic colorectal surgery modifies risk factors for postoperative morbidity. Dis Colon Rectum 2004;47:1686-93.

\section{Endereço para correspondência:}

Geraldo Magela Gomes da Cruz

Rua Rio de Janeiro, 2017/1401

CEP: 30160-042 - Belo Horizonte (MG), Brasil

E-mail: magelacruz@terra.com.br 